\title{
Intercarrier Interference Self-Cancellation Space-Frequency Codes for MIMO-OFDM
}

\author{
Dũng Ngọc Đào, Student Member, IEEE, and Chintha Tellambura, Senior Member, IEEE
}

\begin{abstract}
Space-frequency (SF) codes that exploit both spatial and frequency diversity can be designed using orthogonal frequency division multiplexing (OFDM). However, OFDM is sensitive to frequency offset (FO), which generates intercarrier interference (ICI) among subcarriers. We investigate the pair-wise error probability (PEP) performance of SF codes over quasistatic, frequency selective Rayleigh fading channels with FO. We prove that the conventional SF code design criteria remain valid. The negligible performance loss for small FOs (less than 1\%), however, increases with FO and with signal to noise ratio (SNR). While diversity can be used to mitigate ICI, as FO increases, the PEP does not rapidly decay with SNR. Therefore, we propose a new class of SF codes called ICI self-cancellation SF (ISC-SF) codes to combat ICI effectively even with high FO (10\%). ISC-SF codes are constructed from existing full diversity space-time codes. Importantly, our code design provide a satisfactory tradeoff among error correction ability, ICI reduction and spectral efficiency. Furthermore, we demonstrate that ISC-SF codes can also mitigate the ICI caused by phase noise and time varying channels. Simulation results affirm the theoretical analysis.
\end{abstract}

Index Terms-Inter-carrier interference (ICI), multiple input multiple output (MIMO), orthogonal frequency division multiplexing (OFDM), space-frequency (SF) codes.

\section{INTRODUCTION}

Q PACE-TIME (ST) coding and related multiple input multiple output (MIMO) technologies have rapidly become one of the most active research areas in wireless communications. ST wireless technology applications include third generation mobile and fixed wireless standards [1]. The first ST codes proposed by Tarokh et al. [2] for coherent systems over MIMO quasistatic flat fading channels (i.e., frequency nonselective fading) achieve the maximum diversity order $d=M N$, where $M$ and $N$ are the number of transmit (Tx) and receive (Rx) antennas. Designing ST codes for MIMO frequency selective fading channels has also received much attention. In frequency selective fading channels, the maximum achievable diversity order is $d=\mathrm{LMN}$ where $L$ is the number of paths of the frequency selective fading channels. When ST codes designed for flat fading channels are transmitted over frequency selective fading channels, they can achieve at least the designed diversity order,

Manuscript received July 21, 2004; revised January 11, 2005. The review of this paper was coordinated by Prof. Z. Wang. This work was supported by The National Sciences and Engineering Research Council (NSERC) and Alberta Informatics Circle of Research Excellence (iCORE), Canada. A part of this work has been presented at the Thirty-Eight Asilomar Conference on Signals, Systems and Computers, November 2004, Monterey, CA.

The authors are with Department of Electrical and Computer Engineering, University of Alberta, Edmonton, Alberta T6G 2V4, Canada (e-mail: dndung@ece.ualberta.ca; chintha@ece.ualberta.ca).

Digital Object Identifier 10.1109/TVT.2005.853477 but are not guaranteed to reach the maximum diversity order LMN.

To achieve the full potential diversity order of frequency selective fading channels, in general, ST codes can be designed in the time domain [3] or in the frequency domain using orthogonal frequency division multiplexing (OFDM) and the resulting codes are called space-frequency (SF) codes [4]-[6]. Coding for MIMO-OFDM to achieve high diversity order has drawn much attention after the initial papers [4], [5]. The authors in [7] design SF codes (and also ST codes) using algebraic theory for frequency selective fading channels [8]. [9] introduces a fulldiversity full-rate SF code design, which is developed using complex field coding [10]. The authors in [11] propose a concatenation scheme with Alamouti code [12] as the inner and a trellis code as the outer. However, a simple multipath channel model with equally spaced path delays and uniform power delay profile is assumed in [5]-[7]. Whether these SF coding structures still preserve their diversity order in arbitrary frequency selective fading channels is not proven. More generally, Su et al. [13] derive SF code criteria, showing an explicit relation between the SF code matrix and the characteristic parameters of frequency selective fading channels such as the path delays and power delay profile. The authors in [13] introduce a class of SF codes formed by repetition ST codes. They also show that when any full diversity ST code is used in MIMO-OFDM as an SF code, it achieves at least the diversity order that has been designed in the time domain. Thus, many ST codes are usable as SF codes.

Since the SF codes operate over OFDM, their performance can be affected by underlying impairments such as frequency offset (FO), phase noise, and time varying channels, to name a few. A residual FO exists due to carrier synchronization mismatch and Doppler shift [14]. Residual FO destroys subcarrier orthogonality, which generates intercarrier interference (ICI), and the bit error rate (BER) increases consequently. The effect of such impairments on the conventional (i.e., single input single output (SISO)) OFDM has been widely investigated. For example, in [15], BER is calculated for uncoded SISOOFDM systems with several modulation schemes. The authors in [16], [17] provide BER expressions of MIMO-OFDM employing Alamouti's scheme [12]. The authors in [18] analyze the SF code performance in different propagation settings such as Rayleigh and Rician fading channels and with spatial correlation at the transmitter and/or receiver. However, to the best of our knowledge, the impact of ICI due to FO on the pairwise error probability (PEP) performance of general SF codes, and whether the existing SF code design criteria should be modified when ICI exists, have not been investigated. This important question will be addressed in our paper. 
We analytically show that the conventional SF code design criteria hold even with FO. The performance loss is negligible if the normalized frequency offset (NFO) is small. This loss, however, increases rapidly with the increasing NFO and with signal to noise ratio (SNR). When the NFO is large, the dominance of ICI noise power prevents the typical rapid decay of PEP with SNR and the PEP performance hits a floor.

Since ICI can severely degrade the performance of OFDM, several ICI suppression methods are available (see [19]-[21] and references therein). For SISO-OFDM, the authors in [22], [23] propose ICI self-cancellation coding or polynomial cancellation coding to mitigate ICI (caused by FO) effectively at the cost of reducing spectral efficiency. By analyzing [22], [23] and [13], we derive a new class of SF codes named ICI self-cancellation SF (ISC-SF) codes that provide a satisfactory tradeoff among error correction ability, ICI reduction, and spectral efficiency. ISC-SF codes not only achieve the same diversity order (at least $2 M N$ ) and coding gains as the corresponding SF codes derived in [13], but also notably improve the performance of SF codes with FO. Although our primary focus is the performance of ISC-SF codes with FO, we demonstrate that ISC-SF codes also perform well when ICI is caused by phase noise, and time varying channels. Due to the similar nature of ICI caused by FO, phase noise, and time varying channels, we present the simulation results for FO only.

We summarize the contributions of the paper as follows:

1) The performance of SF codes with FO is analyzed.

2) A new class of SF codes is proposed, which is capable of both error correction and ICI reduction produced by FO, phase noise, and time varying channels.

The rest of the paper is organized as follows. Section II presents the model of MIMO-OFDM systems with FO. Section III reviews the design criteria of SF codes. In Section IV, we derive the PEP performance of SF codes with FO. In Section V, we propose a new class of SF codes that significantly improve the performance of SF codes with FO. Section VI shows how ISC-SF codes also perform well with phase noise and time varying channels. Section VII provides simulations to support the theoretical analysis for the case of FO. The results of the paper are summarized in Section VIII.

Notation: Superscripts $(\cdot)^{T}$ and $(\cdot)^{\dagger}$ denotes matrix transpose and transpose conjugate operations. $E[\cdot]$ denotes statistical mean. $\tilde{H}$ indicates the quantity $H$ being derived with FO. The diagonal matrix with elements of vector $X$ on the main diagonal is denoted by diag $(X)$.

\section{FREQUENCY OFFSET IN MIMO-OFDM SyStEMS}

We now discuss a model of MIMO-OFDM systems and extend this model to include the effects of FO for performance analysis of SF codes with FO.

\section{A. MIMO-OFDM System Model}

Consider a MIMO-OFDM system with $M$ Tx and $N$ Rx antennas as illustrated in Fig. 1. The number of subcarriers in the OFDM modulators is $K$. The $L$-path quasistatic Rayleigh fading channel model is assumed for the link between Tx antenna

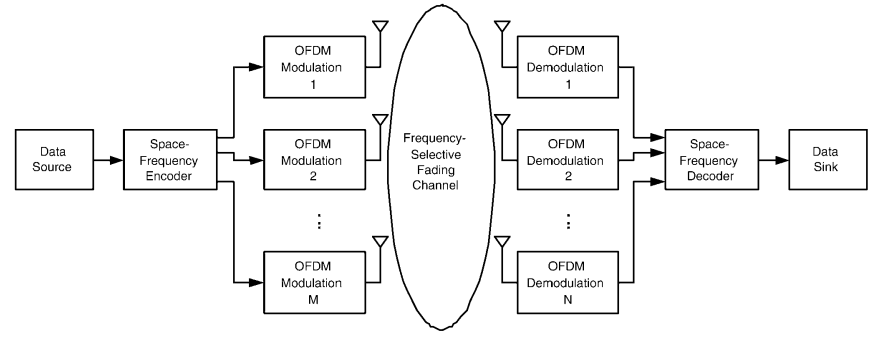

Fig. 1. MIMO-OFDM model.

$m(m=1, \ldots, M)$ and Rx antenna $n(n=1, \ldots, N)$. The channel impulse response in the time domain is [24]

$$
h_{m, n}(t, \tau)=\sum_{l=0}^{L-1} \alpha_{m, n}(t, l) \delta\left(\tau-\tau_{l}\right)
$$

where $\tau_{l}$ is the delay of the $l$ th path $(l=0, \ldots, L-1)$ and $\delta(\cdot)$ denotes Dirac's delta function. The coefficients $\alpha_{m, n}(t, l)$ 's are complex channel gains of the $l$ th path between Tx antenna $m$ and $\mathrm{Rx}$ antenna $n$. They are modeled as zero-mean complex Gaussian random variables (GRV's) with variance $E\left[\left|\alpha_{m, n}(l)\right|^{2}\right]=\delta_{l}^{2}$. We assume the MIMO channel is spatially uncorrelated and remains constant for at least one OFDM symbol duration, but can vary randomly from symbol to symbol. Thus coefficients $\alpha_{m, n}(t, l)$ are independent variables and the time index $t$ can be omitted. Without loss of generality, the total power of $L$-path channels is normalized, so that $\sum_{l=0}^{L-1} \delta_{l}^{2}=1$. The frequency response of the channel between Tx antenna $m$ and Rx antenna $n$ at subcarrier $k$ is

$$
H_{m, n}(k)=\sum_{l=0}^{L-1} \alpha_{m, n}(l) e^{-j 2 \pi k \Delta f \tau_{l}}, \quad j=\sqrt{-1}
$$

where $\Delta f=1 / T_{s}$ is the subcarrier spacing and $T_{s}$ is the OFDM symbol duration.

The transmitted symbols are distributed over $M$ Tx antennas and $K$ subcarriers of each OFDM modulator. Let $c_{m}(k)$ be the $k$ th subcarrier input symbol being sent from Tx antenna $m$ in one OFDM symbol duration. In the frequency domain, the transmitted symbols over $M$ antennas can be represented in the matrix form as follows.

$$
C=\left[\begin{array}{cccc}
c_{1}(0) & c_{2}(0) & \cdots & c_{M}(0) \\
c_{1}(1) & c_{2}(1) & \cdots & c_{M}(1) \\
\cdot & \cdot & \cdot & \cdot \\
c_{1}(K-1) & c_{2}(K-1) & \cdots & c_{M}(K-1)
\end{array}\right] .
$$

Before transmitting, the $K$ symbols of each column in (4) are modulated by inverse discrete Fourier transform (IDFT), and cyclic prefix (CP) symbols are appended [25]. At the receiver side, the $\mathrm{CP}$ symbols are discarded to remove inter-block interference. The remaining $K$ symbols are DFT demodulated to recover transmitted symbols in the frequency domain. Assume that received subcarriers are perfectly sampled and let the received signal at the Rx antenna $n$ be

$$
y_{n}(k)=\sum_{m=1}^{M} c_{m}(k) H_{m, n}(k)+z_{n}(k), \quad k=0, \ldots, K-1,
$$


where $z_{n}(k)$ 's are independent and identically distributed (i.i.d) noise samples, which are modelled as zero-mean complex GRV's. The Tx power from each antenna is normalized to 1 , resulting a noise variance per dimension of $M /(2 \rho)$ where $\rho$ is the average SNR at each Rx antenna.

The input-output relation of MIMO-OFDM systems can be described in several matrix forms. In this paper, we adopt the approach in [13] to derive the PEP of SF codes. For the zero FO case, the received signal in (5) is presented in the vector form as

$$
\mathbf{Y}=\mathbf{D H}+\mathbf{Z}
$$

where $\mathbf{Y}$ is the received signal vector that concatenates received signals of $N \mathrm{Rx}$ antennas as

$$
\begin{aligned}
\mathbf{Y}=\left[y_{1}(0) \ldots y_{1}(K-1) y_{2}(0)\right. & \ldots y_{2}(K-1) \ldots \\
& \left.y_{N}(0) \ldots y_{N}(K-1)\right]^{T},
\end{aligned}
$$

and the channel vector $H$ is of size $K M N \times 1$ is given by

$$
\mathbf{H}=\left[H_{1,1}^{T} \cdots H_{M, 1}^{T} H_{1,2}^{T} \cdots H_{M, 2}^{T} \cdots H_{1, N}^{T} \cdots H_{M, N}^{T}\right]^{T}
$$

where

$$
H_{m, n}=\left[H_{m, n}(0) H_{m, n}(1) \ldots H_{m, n}(K-1)\right]^{T} .
$$

The noise vector $\mathbf{Z}$ is represented similarly to the received vector $\mathbf{Y}$ as

$$
\begin{aligned}
& \mathbf{Z}=\left[z_{1}(0) \ldots z_{1}(K-1) z_{2}(0) \ldots\right. \\
& \left.\quad z_{2}(K-1) \ldots z_{N}(0) \ldots z_{N}(K-1)\right]^{T} .
\end{aligned}
$$

The data matrix $\mathbf{D}$ of size $K M \times K M N$ in (11), shown at bottom of this page, represents the transmitted data in (4). Each matrix $D_{m}$ consists of coded symbols transmitted from antenna $m$,

$$
D_{m}=\operatorname{diag}\left[c_{m}(0), c_{m}(1), \ldots, c_{m}\left(K_{0}-1\right)\right]
$$

\section{B. Model of MIMO-OFDM Systems With FO}

We now extend the MIMO-OFDM system given in (6) for the nonzero FO case. To subsume the FO in (6), we first review the model of SISO systems with FO that was described in [26].

There is always a FO $\delta f$ at the sampling points of received signal in frequency domain [26], [25]. In the SISO-OFDM system, the NFO $\varepsilon$ is defined by $\varepsilon=\delta f / \Delta f$. The NFO is the same for all subcarriers of one OFDM symbol, but may vary from symbol to symbol. In the SISO systems, the received symbol at the $k$ th subcarrier is expressed as follows:

$$
y(k)=\underbrace{S(0) H(k) c(k)}_{\text {desired signal }}+\underbrace{\sum_{p=0, p \neq k}^{K-1} S(p-k) H(p) c(p)}_{\text {ICI }}+z(k)
$$

Let $I(k)$ denotes ICI from the other subcarriers to the received $k$ th subcarrier:

$$
I(k)=\sum_{p=0, p \neq k}^{K-1} S(p-k) H(p) c(p) .
$$

Coefficients $S(k)$ in (14) are expressed as:

$$
S(k)=\frac{\sin [\pi(k+\varepsilon)]}{K \sin \left[\frac{\pi}{K}(k+\varepsilon)\right]} \exp \left[j \pi\left(1-\frac{1}{K}\right)(k+\varepsilon)\right] \text {. }
$$

The coefficient $S(0)$ in (13) can be derived by substituting $k=0$ in (15) to be

$$
S(0)=\frac{\sin (\pi \varepsilon)}{K \sin \left(\frac{\pi}{K} \varepsilon\right)} \exp \left[j \pi\left(1-\frac{1}{K}\right) \varepsilon\right] .
$$

Equations (13) and (16) show that due to the FO, the amplitude of the desired subcarrier is attenuated and its phase is rotated. Furthermore, the ICI from the other subcarriers can be considered as an additional noise. Hence the SNR of the received signal is reduced.

We now generalize (13) for MIMO-OFDM systems and allow for distinct FOs among different $\mathrm{Tx} / \mathrm{Rx}$ antenna pairs. Let the NFO of the transmission link from Tx antenna $m$ and $\mathrm{Rx}$ antenna $n$ be $\varepsilon_{m, n}$. For MIMO systems, the ICI term $I_{n}(k)$ at subcarrier $k$ of each Rx antenna $n$ is the superposition of $M$ ICI terms $I_{m, n}(k)$ caused by transmitted signals from Tx antennas $m$ as

$$
I_{n}(k)=\sum_{m=1}^{M} I_{m, n}(k)
$$

where

$$
I_{m, n}(k)=\sum_{p=0, p \neq k}^{K-1} c_{m}(p) H_{m, n}(p) S_{m, n}(p-k)
$$

and

$$
\begin{aligned}
S_{m, n}(k)= & \frac{\sin \left[\pi\left(k+\varepsilon_{m, n}\right)\right]}{K \sin \left[\frac{\pi}{K}\left(k+\varepsilon_{m, n}\right)\right]} \\
& \times \exp \left[j \pi\left(1-\frac{1}{K}\right)\left(k+\varepsilon_{m, n}\right)\right], \\
S_{m, n}(0)= & \frac{\sin \left(\pi \varepsilon_{m, n}\right)}{K \sin \left(\frac{\pi}{K} \varepsilon_{m, n}\right)} \exp \left[j \pi\left(1-\frac{1}{K}\right) \varepsilon_{m, n}\right] .
\end{aligned}
$$

Equation (13) becomes:

$$
y_{n}(k)=\sum_{m=1}^{M} c_{m}(k) H_{m, n}(k) S_{m, n}(0)+I_{n}(k)+z_{n}(k) .
$$

$$
\mathbf{D}=\left[\begin{array}{ccccccccccccc}
D_{1} & D_{2} & \cdots & D_{M} & 0 & 0 & \cdots & 0 & \cdots & 0 & 0 & \cdots & 0 \\
0 & 0 & \cdots & 0 & D_{1} & D_{2} & \cdots & D_{M} & \cdots & 0 & 0 & \cdots & 0 \\
\vdots & \vdots & \vdots & \vdots & \vdots & \vdots & \vdots & \vdots & \vdots & \vdots & \vdots & \vdots & \vdots \\
0 & 0 & \cdots & 0 & 0 & 0 & \cdots & 0 & \cdots & D_{1} & D_{2} & \cdots & D_{M}
\end{array}\right]
$$


Note that $S_{m, n}(0)$ is a constant with respect to subcarrier index $k$, hence in (21) we can group $H_{m, n}(k)$ and $S_{m, n}(0)$ as:

$$
\tilde{H}_{m, n}(k)=S_{m, n}(0) H_{m, n}(k) .
$$

The equivalent form of (9) is

$$
\tilde{H}_{m, n}=\left[\tilde{H}_{m, n}(0) \tilde{H}_{m, n}(1) \cdots \tilde{H}_{m, n}(K-1)\right]^{T} .
$$

Matrices $\tilde{H}_{m, n}$ are arranged into the matrix $\tilde{\mathbf{H}}$, which has exactly the same structure with the matrix $\mathbf{H}$ given in (8), but the matrix $\tilde{\mathbf{H}}$ accounts for the presence of FO.

The equivalent noise at each received subcarrier is a sum of the ICI noise and complex Gaussian thermal noise terms as

$$
\tilde{z}_{n}(k)=I_{n}(k)+z_{n}(k) .
$$

The MIMO-OFDM model with FO is now written as

$$
\mathbf{Y}=\mathbf{D} \tilde{\mathbf{H}}+\tilde{\mathbf{Z}}
$$

where $\mathbf{Y}$ is the received vector and the matrix $\mathbf{D}$ consists of transmitted symbols. They are described in (7) and (10) accordingly and rewritten in (24) without modification.

The matrix representations (6) and (24) are suitable for deriving the PEP performance of SF codes. In Section III, the PEP upper bound of SF codes without FO based on (6) will be given. It is an asymptotic bound [27] and is tighter than the Chernoff bound [2] at high SNR. In the presence of FO, the equivalent representation (24) will be used to derive the PEP performance (Section IV).

\section{Space-Frequency Code Design CRITERIA}

In the SF encoding process, the source data is twodimensionally encoded across the space (over multiple antennas) and frequency (over the subcarriers of OFDM symbols). An SF codeword may occupy several OFDM symbols [6], [11] or one OFDM symbol [5], [7], [13]. The maximal diversity order can be achieved by coding over the subcarriers of only one OFDM symbol [5], [13], whereas in [6], [11] the maximal diversity order is gained by coding over multiple OFDM symbols, which obviously causes higher coding and decoding delay. We adopt the approach in [13] for our analysis. In the following, we summarize the results of [13].

The input data symbols are divided into $b$-symbol source words and are parsed into blocks and mapped to SF codewords as represented in (4). At the receiver, the maximum likelihood (ML) decoder selects a codeword $E$ if its metric $M e$ is minimum:

$$
M e=\sum_{k=0}^{K-1} \sum_{n=1}^{N}\left|y_{n}(k)-\sum_{m=1}^{M} e_{m}(k) H_{m, n}(k)\right|^{2} .
$$

Assume perfect channel state information (CSI) is available at the receiver but not at the transmitter and perfect symbol timing. The PEP for a transmitted codeword $C$ and erroneously decoded codeword $E$ in a frequency selective fading channel is upper bounded as [13]:

$$
P(C \rightarrow E) \leq\left(\begin{array}{c}
2 \Gamma N-1 \\
\Gamma N
\end{array}\right)\left(\prod_{i=1}^{\Gamma} \lambda_{i}\right)^{-N} \rho^{-\Gamma N}
$$

where $\Gamma$ is the rank of the matrix $Q$ which is defined as

$$
Q \triangleq \Delta o R
$$

and where $\circ$ denotes Hadamard product [28] and $\lambda_{i}(i=$ $1, \ldots, \Gamma)$ are nonzero eigenvalues of $Q$. The matrices $\Delta$ and $R$ are as follows:

$$
\begin{aligned}
\Delta & =(C-E)(C-E)^{\dagger}, \\
R & =R_{m, n}=E\left[H_{m, n} H_{m, n}^{\dagger}\right] \\
& =W \operatorname{diag}\left(\delta_{0}^{2}, \delta_{1}^{2}, \ldots, \delta_{L-1}^{2}\right) W^{\dagger}
\end{aligned}
$$

where

$$
W=\left[\begin{array}{cccc}
1 & 1 & \cdots & 1 \\
w^{\tau_{0}} & w^{\tau_{1}} & \cdots & w^{\tau_{L}-1} \\
\vdots & \vdots & \vdots & \vdots \\
w^{(K-1) \tau_{0}} & w^{(K-1) \tau_{1}} & \cdots & w^{(K-1) \tau_{L-1}}
\end{array}\right]
$$

and $w=e^{-j 2 \pi \Delta f}$

From (26), the SF code design criteria can be stated as follows.

1) Diversity criterion: The minimum rank of $Q$ over all pairs of distinct codewords should be as large as possible.

2) Product criterion: The minimum value of the product $\prod_{i=1}^{\Gamma} \lambda_{i}$ over all pairs of distinct codewords should be also maximized.

From (26), the diversity order of SF codes is $\Gamma N$, maximum achievable diversity order is equal to $\min (L M N, K N)$.

\section{Performance of SF Codes With FO}

We continue the analysis with the two assumptions below:

1) AS1: Residual NFOs $\varepsilon_{m, n}$ are independent of the channel coefficients.

2) AS2: The ICI terms $I_{m, n}(k)$ in (17) and (18) are independent.

The coherent receiver first estimates the channel coefficients. Then the phase shift caused by FO is compensated [26]. Thus the residual FO can be weakly dependent on the channel estimation method. AS1 is given to simplify our analysis. In practice, transmit data over multiple antennas are encoded. There exists a degree of correlation among the transmitted data streams and consequently, the ICI noise terms $I_{m, n}(k)$ are also correlated with respect to the subscript $m$. With AS2, all the ICI noise at the Rx antennas will have the same variance and zero mean. AS2 will be made clearer during the derivation below. Therefore the ML detection in the presence of AWGN noise given in (25) holds.

To investigate the PEP of SF codes with FO using formula (26), the channel coefficients $\tilde{H}_{m, n}(k)$ in (26) should be complex GRVs. This requirement can be met if $S_{m, n}(0)$ is deterministic or NFO is not a random variable (Case 1). In the general case, $\varepsilon_{m, n}$ can be assumed to be i.i.d random variables in the range $\left[E_{1}, E_{2}\right]$, and their values can be changed from OFDM symbol to symbol (Case 2). However, the performance of SF codes with fixed values of FO is of greater interest since it provides a closer look at the performance of SF codes at specific FO values. 
For analytical tractability, we additionally have the third assumption:

3) $\mathrm{AS} 3$ : NFO $\varepsilon_{m, n}$ are constant and the same for all pairs of indices $(m, n): \varepsilon_{m, n}=\varepsilon_{0}$.

If $\left|E_{1}\right|=\left|E_{2}\right|=\left|\varepsilon_{0}\right|$ (if the absolute value of random NFO is not more than a fixed NFO value), we expect that the PEP performance of Case 2 is more optimistic than that of the Case 1 . Therefore, PEP obtained with AS3 is an upper bound of PEP with FO. This assumption will be relaxed in our simulation study (Section VII) and thus more realistic performance evaluation is carried out by simulations. Our analytical results below, however, provide useful insight into the ICI performance of SF codes.

In OFDM systems, $K$ is typically 64 or larger; therefore the central limit theorem can be applied to model the term $I_{m, n}(k)$ as a GRV [25]. The ICI term $I_{n}(k)$ in (17) is a sum of $M$ independent GRVs, it is also a GRV. The first two moments of the term $I_{m, n}(k)$ in (18) by Gaussian approximation are calculated as follows. Assume that coded symbols $c_{m}(p)$ have zero-mean (such as $M$-PAM, $M$-PSK, and $M$-QAM signal constellations), then $E\left[I_{m, n}(k)\right]=0$.

$$
\begin{aligned}
& \text { The variance } \sigma_{I_{m, n}}^{2} \text { of } I_{m, n}(k) \text { in }(18) \text { is } \\
& \begin{aligned}
\sigma_{I_{m}}^{2}= & E\left[\left|I_{m}(k)\right|^{2}\right] \\
= & E\left[\left|\left(\sum_{p=0}^{K-1} c_{m}(p) H_{m, n}(p) S_{m, n}(p-k)\right)\right|^{2}\right] \\
& -E\left[\left|c_{m}(k) H_{m, n}(k) S_{m, n}(0)\right|^{2}\right] \\
= & \sum_{p=0}^{K-1} E\left[\left|c_{m}(p)\right|^{2}\right] E\left[\left|H_{m, n}(p)\right|^{2}\right] E\left[\left|S_{m, n}(p-n)\right|^{2}\right] \\
& -E\left[\left|c_{m}(k)\right|^{2}\right] E\left[\left|H_{m, n}(k)\right|^{2}\right] E\left[\left|S_{m, n}(0)\right|^{2}\right] .
\end{aligned}
\end{aligned}
$$

In the last two rows of (31), the term $E\left[\left|c_{m}(k)\right|^{2}\right]$ is the signal power, which is normalized to 1 . The term $E\left[\left|H_{m, n}(k)\right|^{2}\right]$ is the average of the channel power, and it is also normalized to 1 . Equation (31) becomes

$$
\sigma_{I_{m, n}}^{2}=\sum_{p=0}^{K-1} E\left[|S(p-k)|^{2}\right]-S_{0}
$$

where $S_{0}=E\left[\left|S_{m, n}(0)\right|^{2}\right]=\left|S_{m, n}(0)\right|^{2}$. Note that the residual NFO is usually small, $\varepsilon \leq 0.2$ [25], the number of subcarriers $K \geq 8$, hence $K \sin (\pi \varepsilon / K) \approx \pi \varepsilon$. Let $p(\varepsilon)$ be the probability density function (pdf) of $\varepsilon_{m, n}$. In the case of constant FO, $p(\varepsilon)=1, S_{0}$ can be evaluated as

$$
S_{0}=\left(\frac{\sin \left(\pi \varepsilon_{0}\right)}{\pi \varepsilon_{0}}\right)^{2}=\left[\operatorname{sinc}\left(\varepsilon_{0}\right)\right]^{2} .
$$

It is found in [14] that the sum $\sum_{p=0}^{K-1} E\left[|S(p-k)|^{2}\right]=1$, hence

$$
\sigma_{I_{m, n}}^{2}=1-S_{0} .
$$

It is clear that $\sigma_{I_{m, n}}^{2}$ is independent of indices $m$ and $n$, and it is just dependent on the NFO through $S_{0}$. With AS2, $I_{n}(k)$ is a complex GRV with zero-mean and variance $M\left(1-S_{0}\right)$. Therefore, the ICI noise of MIMO-OFDM $\tilde{z}_{n}(k)$ given in (23) is also a zero-mean complex GRV with variance $\sigma_{z}^{2}=M(1-$ $\left.S_{0}+1 / \rho\right)$. Values of $\sigma_{z}^{2}$ is identical for all $\mathrm{Rx}$ antennas.

From (21), the received signal power is multiplied by $S_{0}$, hence the equivalent SNR at each Rx antenna with FO is

$$
\tilde{\rho}=\frac{M S_{0}}{\sigma_{z}^{2}}=\left(\frac{S_{0}}{\left(1-S_{0}\right) \rho+1}\right) \rho .
$$

Using the MIMO-OFDM model developed in Section II-B and SF code design criteria in Section III, we derive PEP performance given in (26) with FO in the following.

The correlation matrix defined in (29) for equivalent channel matrix $\tilde{H}_{m, n}$ given in (21) has a new form

$$
\begin{aligned}
\tilde{R} & =\tilde{R}_{m, n}=E\left[\tilde{H}_{m, n}\left(\tilde{H}_{m, n}\right)^{\dagger}\right] \\
& =S_{0} E\left[H_{m, n} H_{m, n}^{\dagger}\right]=S_{0} R_{m, n} .
\end{aligned}
$$

Hence the matrix $Q$ in (27) becomes matrix $\tilde{Q}$

$$
\tilde{Q}=\Delta \circ \tilde{R}_{m, n}=S_{0}\left(\Delta \circ R_{m, n}\right)=S_{0} Q .
$$

We can easily verify that:

1) Matrices $Q$ and $\tilde{Q}$ have the same rank $\Gamma$.

2) If $\lambda_{i}$ is an eigenvalue of $Q$, then $\tilde{\lambda}_{i}=S_{0} \lambda_{i}$ is an eigenvalue of $\tilde{Q}$.

Substiting $\tilde{\lambda}=S_{0} \lambda_{i}$ and $\tilde{\rho}$ into (26), and rearranging the terms, the PEP expression with FO is

$$
P(C \rightarrow E) \leq L_{0}\left(\begin{array}{c}
2 \Gamma N-1 \\
\Gamma N
\end{array}\right)\left(\prod_{i=1}^{\Gamma} \lambda_{i}\right)^{-N} \rho^{-\Gamma N}
$$

where

$$
L_{0}=\left(\frac{S_{0}^{2}}{\rho\left(1-S_{0}\right)+1}\right)^{-\Gamma N} .
$$

Comparing (26) and (38), we discern that $L_{0}$ represents the PEP performance loss due to FO. From (26), (38), and (39), we draw the following theoretical conclusions:

1) The design criteria for $S F$ codes without $F O$ is still valid in the case of FO. The code design should maximize the diversity order and coding gain.

2) At the same Tx power, the higher the NFO, the higher the PEP performance loss. That is, at the same PEP, the higher the NFO, the further the PEP curve shifted to the right.

3) The PEP curves will shift right if FO is nonzero. However, with the same NFO, the shift of PEP curves of lower diversity order systems is larger than the shift of PEP curves of the system with higher diversity order. This is due to the fact that given the same loss factor $L_{0}$, the SNR compensation for this loss is smaller for the codes with higher diversity order [cf. (39)]. Thus, the higher diversity order systems are more robust to the effects of FO.

4) If $\rho\left(1-S_{0}\right) \gg 1$ or at high Tx power and high value of FO,

$P(C \rightarrow E) \leq\left(\begin{array}{l}2 \Gamma N-1 \\ \Gamma N\end{array}\right)\left(\prod_{i=1}^{\Gamma} \lambda_{i}\right)^{-N} \times\left(\frac{S_{0}^{2}}{1-S_{0}}\right)^{-\Gamma N}$ 
The PEP is no longer inversely proportional with the SNR and hits a floor.

These analytical results can be anticipated since the ICI term is considered as an additional Gaussian noise. When FO is small, the ICI power is smaller than the power of thermal noise; thus its impact on the performance of SF codes is negligible. However, when the FO is large, the ICI noise dominates thermal noise. The ICI power increases with desired signal power; therefore when SNR is large, ICI causes the error floor as we have derived. Nevertheless, the analytical results reveal explicitly that when residual FO is small, about $1 \%$, the performance loss is almost negligible (see Section VII).

To complete this section, we note that one can derive the ML receiver using (21) and (25), and the same result as (38) can be obtained.

\section{ICI SELF-CANCELLATION SF CODES}

In Section IV, we have shown that if the NFO is high, the PEP performance is limited by a floor level at high SNR. Thus a SF code which can mitigate the effects of high NFO is desirable. We now relate SF codes and polynomial cancellation coding (PCC). PCC is first proposed by authors in [22]. This idea is further analyzed in [23] from theory of finite differences.

We now summarize and analyze the main results of PCC in [22]. To mitigate the ICI caused by FO, one coded or uncoded data symbol modulates a group of $r$, where $r=2,3,4, \ldots$, consecutive subcarriers. The optimum weighting coefficients for $r$ subcarriers to minimize ICI are the coefficients of the polynomial $(1-D)^{r-1}$. The code rate of PCC is $1 / r$. The ICI performance of this coding scheme increases with $r$ at the cost of spectral efficiency. Simulation results [22] show that this coding scheme with $r=2$ (or code rate $1 / 2$ ) outperforms the system using rate $1 / 2$ convolutional code when NFO is high (20\%), but PCC performs poorly when NFO is small or medium $(\leq 10 \%)$. The reason is that this code is particularly designed to minimize ICI and hence may not be suitable for error correction purposes. To improve the performance of PCC, an outer error control code is required. The resultant concatenated code has lower rates as $r$ increases. Thus, the smallest possible value of $r, r=2$ is of practical interest.

Another result of [22] is the ICI cancelling demodulation (ICD) concept. For example, when $r=2$, one data symbol $x$ is sent over two subcarriers that satisfies: $c(k)=x, c(k+1)=$ $-x$. This process is called ICI cancellation modulation (ICM). The received signals $y(k)$ and $y(k+1)$ create a new signal for detection: $y^{*}(k)=y(k)-y(k+1)$. This process is named ICD. The combination of ICM and ICD is called ICI selfcancellation (ISC). The ICI noise power of ISC is smaller than the original ICI and ICI of ICM, therefore the ISC cancellation demodulation is powerful against FO. From a diversity point of view, using two values $y(k)$ and $y(k+1)$ to detect one transmitted symbol $x$ could yield a diversity order of two. Since there is a strong correlation between adjacent subcarriers, however, the use of the two signals $y(k)$ and $y(k+1)$ may not in fact provide a diversity order of two. Our target is to maximize the diversity order of SF codes; thus, in this paper, the ICM is our concern, but not the ISC (the combination of ICM and ICD).

In summary, PCC is suitable for ICI reduction. However, its error correction ability and spectral efficiency are low. Therefore, a low order PCC code with $r=2$ concatenated with powerful error control codes would be a good tradeoff solution. We next develop the idea of ICM to design a class of SF codes that are robust to ICI.

Su et al. [13] show that the SF code formed by repeating each row of a full diversity order ST codeword $r$ times $(1 \leq r \leq L)$ achieves at least the diversity order $d=r M N$. This repetition obviously reduces the spectral efficiency; thus we consider only $r=2$.

Let the number of OFDM subcarriers $K=2 \hat{K}$. Suppose that the length of an SF codeword equals the number of subcarriers $K$. If the SF codeword length is smaller than $K$, a zero-padding matrix can be used for the remaining subcarriers. An SF codeword has the form

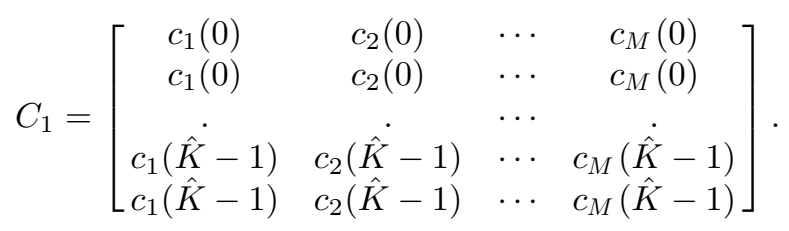

Applying the ICM scheme, for $r=2$, this scheme is actually a repetition scheme but the repeated symbols are sign-reversed. In the case of MIMO-OFDM, the repeated rows are sign-reversed to form new ISC-SF codewords as

$$
C_{2}=\left[\begin{array}{cccc}
c_{1}(0) & c_{2}(0) & \cdots & c_{M}(0) \\
-c_{1}(0) & -c_{2}(0) & \cdots & -c_{M}(0) \\
\cdot & \dot{K} & \cdots & \dot{\hat{K}}-1) \\
c_{1}(\hat{K}-1) & c_{2}(\hat{K}-1) & \cdots & c_{M}(\hat{K}-1) \\
-c_{1}(\hat{K}-1) & -c_{2}(\hat{K}-1) & \cdots & -c_{M}(\hat{K}-1)
\end{array}\right] .
$$

We call the SF coding schemes given in (41) and (42) SC1 and $\mathrm{SC} 2$ for short. We now prove that the new coding scheme $\mathrm{SC} 2$ yields the same coding gain and diversity order (at least $d=2 M N$ ) compared with $\mathrm{SC} 1$, but $\mathrm{SC} 2$ integrates ICI selfcancellation capability.

Proof: Consider an entry $a_{i, j}$ of the matrix $\Delta_{1}$ defined in (28) being created by SF codewords (41). The entry $b_{i, j}$ of the matrix $\Delta_{2}$ being created by SF codewords in (42) is related with $a_{i, j}$ as

$$
b_{i j}= \begin{cases}a_{i j}, & \text { if }(i+j) \text { is even } \\ -a_{i j}, & \text { if }(i+j) \text { is odd. }\end{cases}
$$

Note that the size of $\Delta_{1}$ and $\Delta_{2}$ is $K \times K, K=2 \hat{K}$ and, in particular, they can be written as follows:

$$
\Delta_{1}=\left[\begin{array}{cccc}
a_{11} & a_{12} & \cdots & a_{1 K} \\
a_{21} & a_{22} & \cdots & a_{2 K} \\
\vdots & \vdots & \ddots & \vdots \\
a_{K 1} & a_{K 2} & \cdots & a_{K K}
\end{array}\right]
$$




$$
\begin{aligned}
\Delta_{2} & =\left[\begin{array}{cccc}
b_{11} & b_{12} & \cdots & b_{1 K} \\
b_{21} & b_{22} & \cdots & b_{2 K} \\
\vdots & \vdots & \ddots & \vdots \\
b_{K 1} & b_{K 2} & \cdots & b_{K K}
\end{array}\right] \\
& =\left[\begin{array}{cccc}
+a_{11} & -a_{12} & \cdots & -a_{1 K} \\
-a_{21} & +a_{22} & \cdots & +a_{2 K} \\
\vdots & \vdots & \ddots & \vdots \\
-a_{K 1} & +a_{K 2} & \cdots & +a_{K K}
\end{array}\right] .
\end{aligned}
$$

The matrix $R$ defined in (29) is the same for both SC1 and SC2. Therefore, comparing the signs of entries of $Q_{1}=\Delta_{1} \circ R$ and $Q_{2}=\Delta_{2} \circ R$ (defined in (27)), we can see that the signs of entries of $Q_{2}$ are changed in accordance with the sign changes of entries of $\Delta_{2}$ compared to $\Delta_{1}$. Thus we have following relationship:

1) If vector $X_{1}=\left[\begin{array}{lllllll}x_{1} & x_{2} & x_{3} & x_{4} & \cdots & x_{K-1} & x_{K}\end{array}\right]$ is an eigenvector of $Q_{1}=\Delta_{1} \circ R$, then $X_{2}=$ $\left[\begin{array}{lllllll}x_{1} & -x_{2} & x_{3} & -x_{4} & \cdots & x_{K-1} & -x_{K}\end{array}\right]$ is an eigenvector of $Q_{2}=\Delta_{2} \circ R$ and vice versa, where $\left(Q_{1}, \Delta_{1}\right)$ and $\left(Q_{2}, \Delta_{2}\right)$ are sets of matrices associated with $\mathrm{SF}$ codewords defined in (27) and (28) respectively.

2) If $\lambda$ is an eigenvalue of $X_{1}$, it is also an eigenvalue of $X_{2}$ and vice versa. The rank of $Q_{1}$ and $Q_{2}$ are the same hence $\mathrm{SF}$ codes $\mathrm{SC} 1$ and $\mathrm{SC} 2$ have the same diversity order.

Thus SF codes constructed as in (41) and (42) have the same diversity order and coding gain.

We refer to SC2 codes as ICI self-cancellation SF codes or ISC-SF codes for short. The code rate of ISC-SF is $R_{t} / 2$, where $R_{t}$ is the code rate of the underlying ST code. Repetition the $\mathrm{SF}$ codewords more than twice in combination with polynomial cancellation coding will gain additional diversity order and ICI mitigation. However, the price paid for those improvements is the spectral efficiency reduction. Moreover, from the simulations in Section VII, we will see that the ISC-SF codes $(r=2)$ perform well compared with the codes without ICI cancellation. The higher order PCC codes $(r>2)$ would not significantly improve ICI reduction. Thus analysis of the SF schemes with higher repetition orders (i.e., lower rate) is not discussed further. The ISC-SF coding scheme (42) gives a satisfactory tradeoff among error control performance, ICI reduction and spectral efficiency.

\section{Phase Noise AND Time VARying Channel}

Channel variations and phase noise also produce ICI. In Section II, we assume that the MIMO channels remain constant during one OFDM symbol. However, due to the relative movement of the transmitter and receiver, the channels may vary during one OFDM symbol. This variation causes ICI and makes BER increase (see [29], [21], and references therein). For the description of phase noise and its effects, refer to [30], [19], [31].

In the following, we use a continuous time model to show how ICI is created by phase noise and time varying channels. Consider SISO-OFDM systems. The transmitted signal over a block, including $\mathrm{CP}$, is given by

$$
s(t)=\sum_{k=0}^{K-1} c(k) e^{j 2 \pi k \Delta f t}, \quad-T_{c p} \leq t \leq T_{s},
$$

where $T_{c p}$ is the length of CP. The duration of one OFDM symbol with $\mathrm{CP}$ is $T_{b}=T_{c p}+T_{s}$.

The signal at the input of the receiver is

$$
\begin{aligned}
r(t) & =s(t) * h(t, \tau)=\int_{-\infty}^{\infty} h(t, \tau) s(t-\tau) d t+n(t) \\
& =\sum_{k=0}^{K-1} \sum_{l=0}^{L-1} c(k) h\left(t, \tau_{l}\right) e^{-j 2 \pi k \Delta f \tau_{l}} e^{j 2 \pi k \Delta f t}+n(t) .
\end{aligned}
$$

where $n(t)$ is an AWGN process with zero-mean and onesided power spectral density (PSD) is $N_{0}$. At the demodulator, the phase noise $\phi(t)$ between the carrier and the local oscillator is added to the phase of received signals. In the baseband representation, adding phase noise is equivalent to multiplying $r(t)$ with $\theta(t)=e^{j \phi(t)}$.

If we consider the effect of FO only as in Section II-B, let $\phi(t)=0$ and $h(t, \tau)$ be constant, then one can derive $y(p)$ as in (13). In this section, we consider the ICI due to phase noise and time varying channels, thus we let $\delta f=0$ to simplify the expressions. The demodulated signal $y(p)$ is

$$
\begin{aligned}
y(p)= & T_{s}^{-1} \sum_{k=0}^{K-1} \sum_{l=0}^{L-1} c(k) e^{-j 2 \pi k \Delta f \tau_{l}} \\
& \times \int_{0}^{T_{s}} h\left(t, \tau_{l}\right) e^{j \phi(t)} e^{j 2 \pi(k-p) \Delta f t} d t+n(p) .
\end{aligned}
$$

To consider the effect of time varying channels only, let $\phi(t)=0$ in (45); we then have

$$
y(p)=\underbrace{\breve{H}(p) c(p)}_{\text {desired signal }}+\underbrace{\sum_{k=0, k \neq p}^{K-1} \breve{H}(p-k) c(k)}_{\text {ICI }}+n(p)
$$

where

$$
\breve{H}(i)=\sum_{l=0}^{L-1}\left(c(k) e^{-j 2 \pi k \Delta f \tau_{l}} \int_{0}^{T_{s}} h\left(t, \tau_{l}\right) e^{j 2 \pi(k-p) \Delta f t} d t\right) .
$$

The ICI term in (46) can be approximated by a zero-mean GRV. Its power can be found in [32].

If we consider the effect of phase noise only, let $h(t, \tau)$ in (45) be constant, and $y(p)$ becomes

$$
y(p)=\underbrace{a(0) H(k) c(p)}_{\text {desired signal }}+\underbrace{\sum_{k=0, k \neq p}^{K-1} a(p-k) H(k) c(k)}_{\text {ICI }}+n(p)
$$




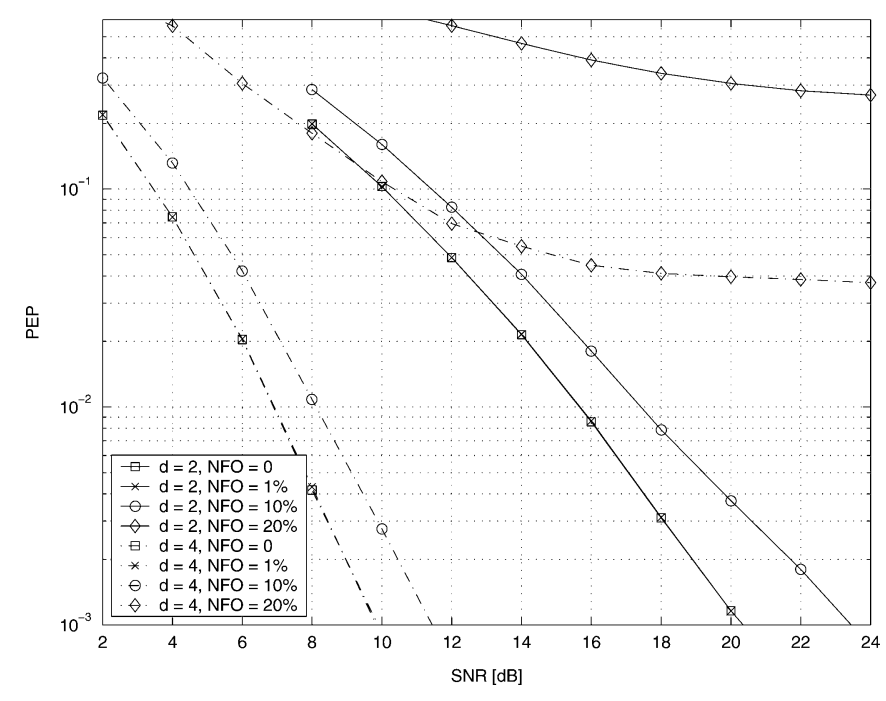

Fig. 2. Performance of SF codes with $K=64$, constant FO for the six path COST207 typical urban channel model.

where

$a(i)=T_{s}^{-1} \int_{0}^{T_{s}} \theta(t) e^{-j 2 \pi i \Delta f t} d t, \quad a(0)=T_{s}^{-1} \int_{0}^{T_{s}} \theta(t) d t$.

Since $\breve{H}(i)$ in (47) is a non-GRV and $a(0)$ in (49) is nonconstant, analyzing the PEP of SF codes with phase noise and time varying channels becomes difficult. However, comparing (46) and (48) with (14), one can see the ICI contributions of subcarriers to one subcarrier due to time varying channels and phase noise are similar to the ICI contribution due to FO. Thus, ISC-SF codes with capability of cancelling ICI should perform well compared with the SF codes without this feature in cases of phase noise and time varying channels, as discussed for the case of FO.

\section{SIMULATION RESULTS AND DisCUSSION}

We give simulation results to verify the theoretical analysis in Sections IV and V for SF codes with FO. We use two channel models: 1) a simple two path channel with uniform power delay profile and time delay between the two paths is $5 \mu \mathrm{s}$; and 2) the sixpath COST207 typical urban (COST207 TU) channel model [24], a more realistic model. We use algebraic ST convolutional codes [7], [33] of code rate $1 / 2$ with generator polynomial (5, 7) [34] without channel interleaver. The Viterbi decoder [33], [34] is employed. The similar simulation results are observed for both channel models. Thus, we present the simulations with, COST207 TU channel model for brevity.

\section{A. Simulations With Constant FO}

We compare the performance of SF codes for $1 \%, 10 \%$, and $20 \%$ NFO. Fig. 2 illustrates PEP curves of two OFDM systems with 64 subcarriers and two Tx antennas. System 1 is equipped with one Rx antenna and System 2 has two Rx antennas, so that the diversity order of the two systems is at least $d=2$ and $d=4$,

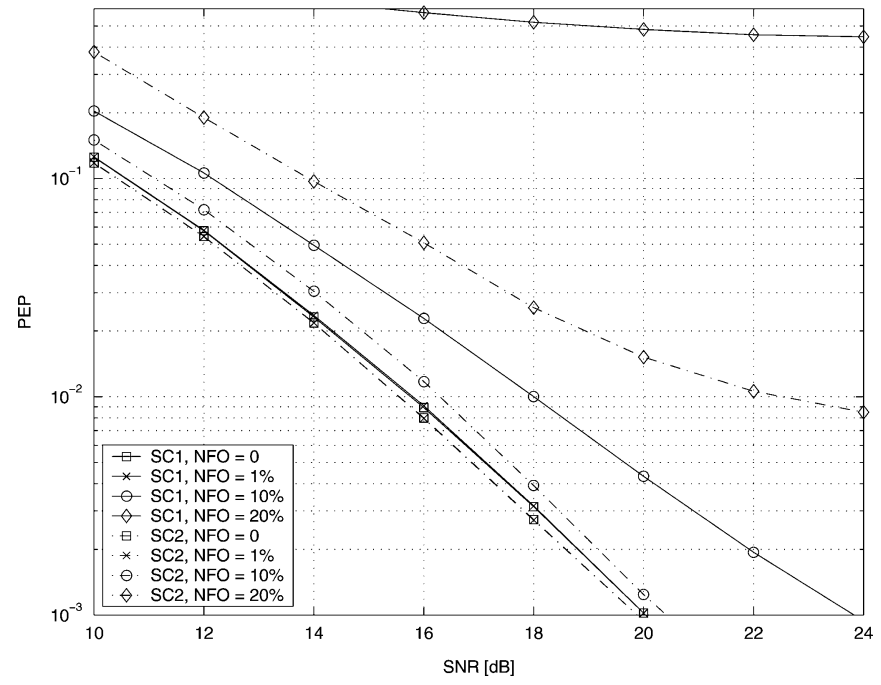

Fig. 3. Performance of SF codes with $K=128, r=2$, constant FO, six path COST207 channel model with and without ICI self-cancellation.

respectively. Using (33), for $1 \% \mathrm{NFO}, S_{0}=0.9997$, and $L_{0} \approx 1$ in the SNR region of interest $(\leq 30 \mathrm{~dB})$. Therefore, theoretically, the performance loss is not significant. Fig. 2 confirms this conclusion. If the NFO is small, say $1 \%$, the PEP curves almost overlap the PEP of the systems with no FO. In case NFO is $10 \%$, the PEP curves of all systems are shifted to the right and are less steeper than the curves of PEP with $1 \%$ NFO; this shift is larger for the system with smaller diversity order. For example, at PEP $=10^{-3}$, the PEP curve of the system with $d=2$ shifts right $1.4 \mathrm{~dB}$, whereas it is $0.8 \mathrm{~dB}$ for the system with $d=4$. The SNR needed to compensate for the effect of FO increases with NFO. When NFO is $20 \%$, the PEP reduces slightly even if SNR increases significantly. The PEP performance reaches a floor at an SNR of about $22 \mathrm{~dB}$. This symptom is more serious for low diversity order systems, where the floor level is higher than that of higher diversity order systems.

\section{B. Simulations With ICI Self-Cancelation SF Codes}

The performance of SF coding schemes with and without ICI self-cancellation ( $\mathrm{SC} 2$ and $\mathrm{SC} 1$ accordingly) is illustrated in Fig. 3. The systems to be examined have 128 subcarriers, $2 \mathrm{Tx}$ and $1 \mathrm{Rx}$ antennas. We can verify the results in Section V that when FO is absent, $\mathrm{SC} 1$ and $\mathrm{SC} 2$ have the same diversity order. The difference between the coding gains of the two schemes is very small, less than $0.2 \mathrm{~dB}$ at the plotted SNR. This difference is expected to be zero at the higher SNR region. Once again, the performance loss for $1 \% \mathrm{NFO}$ is negligible for both $\mathrm{SC} 1$ and SC2. However, the improvement of SC2 over SC1 is remarkable when NFO is $10 \%$ and $20 \%$. For example, in Fig. 3 at PEP $=10^{-3}$ and $10 \% \mathrm{NFO}$, the performance loss of SC1 is about $3.7 \mathrm{~dB}$, whereas the loss is about $0.5 \mathrm{~dB}$ with $\mathrm{SC} 2$. This improvement is significant in Fig. 3 where the loss of SC2 is only $0.5 \mathrm{~dB}$. In addition, $\mathrm{SC} 2$ lowers the error floor level notably when NFO is very high at $20 \%$. 


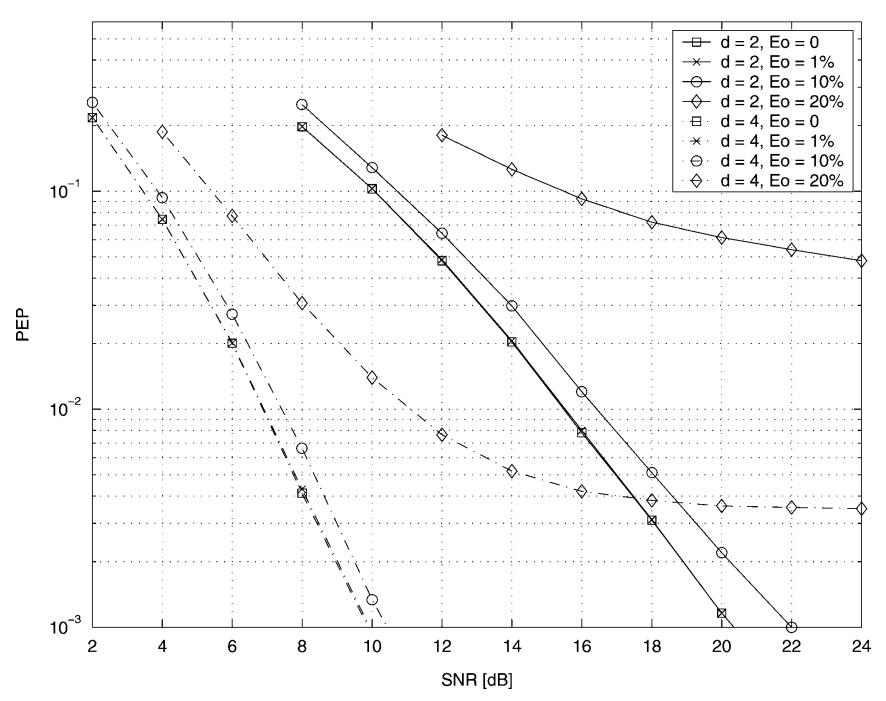

Fig. 4. Performance of SF codes with $K=64$, uniformly distributed FO and the six path COST207 typical urban channel model.

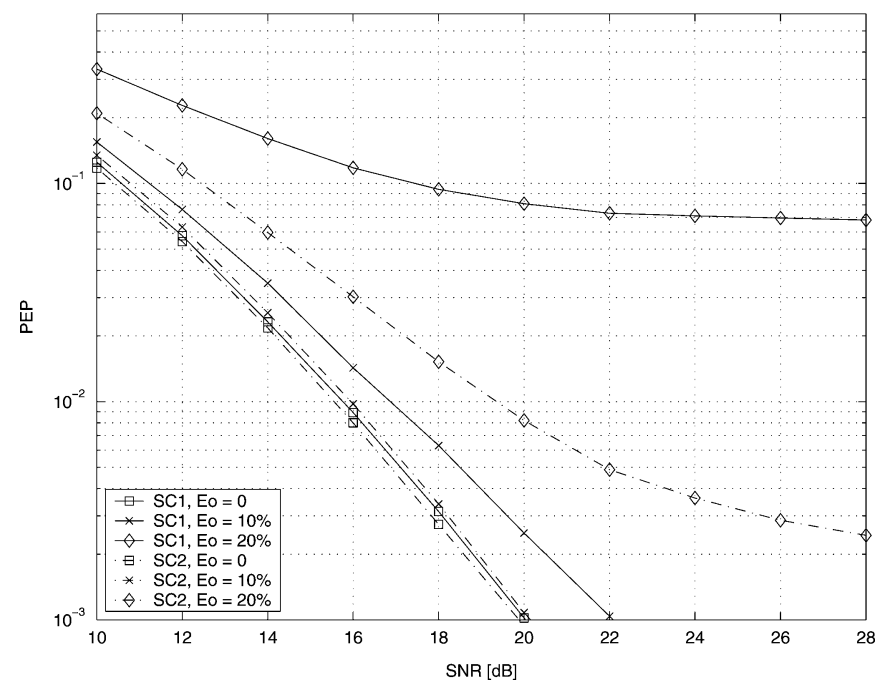

Fig. 5. Performance of SF codes with $K=128, r=2$, uniformly distributed FO, six path COST207 channel model with and without ICI self-cancellation.

\section{Simulations With Variable FO}

In practice, the $\mathrm{FO}$ values of $\mathrm{Rx}$ signals at antenna $n$ that were transmitted from antenna $m$ can be different, and they vary from symbol to symbol. We now provide simulation results for variable FO. The distribution of NFO values are assumed uniform over the range $\left[-E_{0}, E_{0}\right]$, where $\left|E_{0}\right|$ is the maximum NFO. Similar to the previous simulations, we will examine performance of the SF codes for $\left|E_{0}\right|=1 \%, 10 \%$, and $20 \%$.

As discussed in Section IV, assumption AS3, the performance of SF codes with variable FO should be upperbounded by the performance curves with the constant FO. Fig. 4 presents performance of 64-subcarrier systems with one and two Tx antennas. The two systems have two Tx antennas. By examining Figs. 2 and 4, exactly the same observations can be made as with the constant FO. The only difference between the constant and variable FO cases is that in the latter case, the performance loss is always less than the loss of the former case, as expected. For example, comparing Figs. 2 and 4, for the system $1(d=2)$, $10 \% \mathrm{NFO}$, at PEP $=10^{-3}$, the loss is about $3 \mathrm{~dB}$ in case of constant $\mathrm{FO}$, while it is about $1.7 \mathrm{~dB}$ for the system with varying FO.

Fig. 5 presents the performance of the SF coding schemes SC1 and SC2 with variable FO. Comparing their performances that are given in Fig. 3 with fixed FO, the loss for variable FO is smaller.

\section{CONCLUSION}

We developed a MIMO-OFDM model with FO and used this model to analyze the PEP performance of SF codes. Using the PEP upper bound of SF codes, we showed that the conventional code design criteria remain valid provided FO is small. ICI is less severe for SF codes with high diversity order. Therefore, diversity not only improves the performance of OFDM systems in the dispersive channels, but also makes the system robust to ICI. Furthermore, we proposed a new class of SF codes, ISC-SF codes with diversity order of at least $2 M N$. ISC-SF codes are constructed from ST codes to efficiently mitigate ICI caused by FO, phase noise, and time varying channels. This class of SF codes permits a good tradeoff among error correction capability, ICI reduction, and spectral efficiency. Our results suggest a new direction in the design of ST/SF codes capable of both error correction and ICI reduction.

\section{REFERENCES}

[1] S. N. Diggavi, N. Al-Dhahir, A. Stamoulis, and A. R. Calderbank, "Great expectations: the value of spatial diversity in wireless networks," Proc. IEEE, vol. 92, pp. 219-270, Feb. 2004.

[2] V. Tarokh, N. Seshadri, and A. R. Calderbank, "Space-time codes for high data rate wireless communication: performance analysis and code construction," IEEE Trans. Inf. Theory., vol. 44, pp. 744-765, Mar. 1998.

[3] M. Qin and R. Blum, "Properties of space-time codes for frequency selective channels," IEEE Trans. Signal Process, vol. 52, pp. 694-702, Mar. 2004.

[4] B. Lu and X. Wang, "Space-time code design in OFDM systems," in Proc. IEEE GLOBECOM, San Francisco, CA, Nov. 2000, pp. 1000-1004.

[5] H. Bolcskei and A. J. Paulraj, "Space-frequency coded broadband OFDM systems," in Proc. IEEE Wireless Communications Networking Conf. (WCNC), Chicago, IL, vol.1, Sep. 2000, pp. 1-6.

[6] Z. Liu, Y. Xin, and G. B. Giannakis, "Space-time-frequency coded OFDM over frequency selective fading channels," IEEE Trans. Signal Process, vol. 50, pp. 2465-2476, Oct. 2002.

[7] H. El Gamal, A. R. Hammons Jr., Y. Liu, M. P. Fitz, and O. Y. Takeshita, "On the design of space-time and space-frequency codes for MIMO frequency selective fading channels," IEEE Trans. Inf. Theory., vol. 49, pp. 2277-2292, Sep. 2003.

[8] H. E. Gamal and A. R. Hammons Jr., "On the design of algebraic spacetime codes for MIMO block-fading channels," IEEE Trans. Inf. Theory., vol. 49, pp. 151-163, Jan. 2003.

[9] X. Ma and G. B. Giannakis, "Full-diversity full-rate complex-field spacetime coding," IEEE Trans. Signal Process, vol. 51, pp. 2917-2930, Nov. 2003.

[10] Z. Wang and G. B. Giannakis, "Complex-field coding for OFDM over fading wireless channels," IEEE Trans. Inf. Theory., vol. 49, pp. 707-720, Mar. 2003.

[11] Y. Gong and K. B. Letaief, "An efficient space-frequency coded OFDM system for broadband wireless communications," IEEE Trans. Commun., vol. 51, pp. 2019-2029, Dec. 2003.

[12] S. Alamouti, "A simple transmit diversity technique for wireless communications," IEEE J. Sel. Areas. Commun., vol. 16, pp. 1451-1458, Oct. 1998. 
[13] W. Su, Z. Safar, M. Olfat, and K. J. R. Liu, "Obtaining full-diversity space-frequency codes from space-time codes via mapping," IEEE Trans. Signal Process, vol. 51, pp. 2905-2916, Nov. 2003.

[14] T. Pollet, M. V. Bladel, and M. Moeneclaey, "BER sensitivity of OFDM systems to carrier frequency offset and wiener phase noise," IEEE Trans. Commun., vol. 43, no. 2/3/4, pp. 191-193, Feb./Mar./Apr. 1995.

[15] K. Sathananthan and C. Tellambura, "Probability of error calculation of OFDM systems with frequency offset," IEEE Trans. Commun., vol. 49, pp. 1884-1888, Nov. 2001.

[16] H.-K. Song, S.-J. Kang, M.-J. Kim, and Y.-H. You, "Error performance analysis of STBC-OFDM systems with parameter imbalances," IEEE Trans. Broadcast., vol. 50, pp. 76-82, Mar. 2004.

[17] R. Narasimhan, "Performance of diversity schemes for OFDM systems with frequency offset, phase noise, and channel estimation errors," IEEE Trans. Commun., vol. 50, pp. 1561-1565, Oct. 2002.

[18] H. Bolcskei, M. Borgmann, and A. J. Paulraj, "Impact of the propagation environment on the performance of space-frequency coded MIMOOFDM,” IEEE J. Sel. Areas. Commun., vol. 21, pp. 427-439, Apr. 2003.

[19] L. Piazzo and P. Mandarini, "Analysis of phase noise effects in OFDM modems," IEEE Trans. Commun., vol. 50, pp. 1696-1705, Oct. 2002.

[20] J. Armstrong, "Analysis of new and existing methods of reducing intercarrier interference due to carrier frequency offset in OFDM," IEEE Trans. Commun., vol. 47, pp. 365-369, Mar. 1999.

[21] X. Cai and G. B. Giannakis, "Bounding performance and suppressing intercarrier interference in wireless mobile OFDM,"IEEE Trans. Commun., vol. 51, pp. 2047-2056, Dec. 2003.

[22] Y. Zhao and S. Häggman, "Intercarrier interference self-cancellation scheme for OFDM mobile communication systems," IEEE Trans. Commun., vol. 49, pp. 1185-1191, Jul. 2001.

[23] K. A. Seaton and J. Armstrong, "Polynomial cancellation coding and finite differences," IEEE Trans. Inf. Theory, vol. 46, no. 1, pp. 311-313, Jan. 2000.

[24] G. L. Stuber, Principles of Mobile Communications, 2nd ed., Norwell, MA: Kluwer, 2001.

[25] T. Keller and L. Hanzo, "Adaptive multicarrier modulation: A convenient framework for time-frequency processing in wireless communications," IEEE Proc., vol. 88, pp. 611-640, May 2000.

[26] P. H. Moose, "A technique for orthogonal frequency division multiplexing frequency offset correction," IEEE Trans. Commun., vol. 42, no. 10 , pp. 2908-2914, Oct. 1994.

[27] S. Siwamogsatham, M. P. Fitz, and J. H. Grimm, "A new view of performance analysis of transmit diversity schemes in correlated Rayleigh fading," IEEE Trans. Inf. Theory, vol. 48, pp. 950-956, Apr. 2002.

[28] R. A. Horn and C. R. Johnson, Matrix Analysis. Cambridge, U.K.: Cambridge Univ. Press, 1985.

[29] M. Russell and G. L. Stuber, "Interchannel interference analysis of OFDM in a mobile environment," in Proc. IEEE Vehicular Technology Conf. (VTC), Chicago, IL, 1995, pp. 820-824.

[30] P. Robertson and S. Kaiser, "Analysis of the effect of phase-noise in orthogonal frequency division multiplex (OFDM) systems," in Proc. IEEE Int. Conf. Communications (ICC), Seattle, WA, 1995, pp. 1652-1657.
[31] R. Van Nee and R. Prasad, OFDM for Wireless Multimedia Communications. Boston/London: Artech House, 2000.

[32] Y. Li and L. J. Cimini Jr., "Bounds on the interchannel interference of OFDM in time-varying impairments," IEEE Trans. Commun., vol. 49, pp. 401-404, Mar. 2001.

[33] A. R. Hammons Jr. and H. El Gamal, "On the theory of space-time codes for PSK modulation," IEEE Trans. Inf. Theory., vol. 46, pp. 524-542, Mar. 2000.

[34] J. G. Proakis, Digital Communications, 4th ed., New York: McGraw-Hill, 2001 .

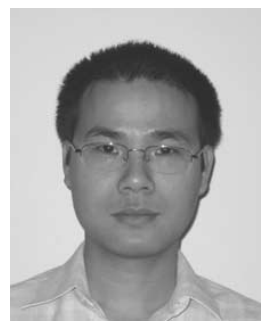

Dũng Ngoc Đào (S’02) received the B.Eng. degree in communications engineering from Hanoi University of Technology, Hanoi, Vietnam, in 1995 and the M.Eng. degree in telecommunications from Asian Institute of Technology (AIT), Thailand, in 2002. He was with Siemens Information and Communications Networks Vietnam and held various technical positions from 1995 to 2001 . He is currently working toward the Ph.D. degree in the Department of Electrical and Computer Engineering, University of $\mathrm{Al}-$ berta, Canada. His research interests include spacetime coding, multicarrier transmission, and error control coding.

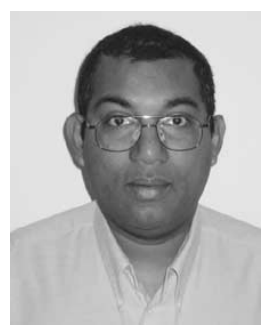

Chintha Tellambura (SM'02) received the B.Sc. degree (with first-class honors) from the University of Moratuwa, Moratuwa, Sri Lanka, in 1986, the M.Sc. degree in electronics from the University of London, London, U.K., in 1988, and the Ph.D. degree in electrical engineering from the University of Victoria, Victoria, BC, Canada, in 1993.

He was a Postdoctoral Research Fellow with the University of Victoria (1993-1994) and the University of Bradford (1995-1996). He was with Monash University, Melbourne, Australia, from 1997 to 2002. Presently, he is an Associate Professor with the Department of Electrical and Computer Engineering, University of Alberta. His research interests include coding, communication theory, modulation, equalization, and wireless communications

Prof. Tellambura is an Associate Editor for both the IEEE Transactions on Communications and the IEEE Transactions on Wireless Communications. $\mathrm{He}$ is a Co-Chair of the Communication Theory Symposium in Globecom'05 to be held in St. Louis, MO. 